\title{
Intermediate osteotomies in rhinoplasty: a new perspective
}

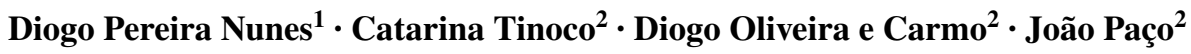

Received: 17 December 2016 / Accepted: 14 March 2017

(C) Springer-Verlag Berlin Heidelberg 2017

\begin{abstract}
The nasal dorsum framework plays a major role in nasal and facial harmony. This study presents a new approach to an already known technique, intermediate osteotomies, which can be used routinely in patients with cosmetically unpleasant changes in eyebrow-tip line features, allowing the acquisition of a natural, aesthetically agreeable and harmonious dorsum contour, while preserving nasal function. The approach was evaluated retrospectively in 50 consecutive patients at a tertiary referral hospital. Patients were asked to complete a survey to rate their satisfaction with the cosmetic outcome on a 5-point visual analog scale (VAS) and to compare the impact of nasal obstruction on their quality of life, pre- and postoperatively, by completing the validated Nasal Obstruction Symptom Evaluation (NOSE) scale. Satisfaction was scored as excellent (satisfaction level VAS average score, 4.4), with more than $50 \%$ of the patients completely satisfied with the aesthetic result, while NOSE scale scores demonstrated preservation of nasal function postoperatively. Typical complications of the upper third of the nose following osteotomies were not observed.
\end{abstract}

Keywords Rhinoplasty $\cdot$ Osteotomies $\cdot$ Intermediate osteotomies $\cdot$ Nasal vault

Diogo Pereira Nunes

pereiranunes.diogo@gmail.com

1 Department of Otolaryngology, Head and Neck Surgery, Klinikum Bremen-Mitte, St.-Jürgen-Str. 1, 28177 Bremen, Germany

2 Department of Otolaryngology, Head and Neck Surgery, CUF Infante Santo Hospital, Lisbon, Portugal

\section{Introduction}

Nasal osteotomies continue to be some of the most challenging techniques in facial plastic surgery, as in most cases they require extensive surgical experience and visionless tissue manipulation to achieve facial symmetry and harmony.

Osteotomies are used to narrow a wide bony vault, to widen a narrow bony vault, to correct a deviated nose or lateral nasal wall deformities, or to close an open-roof. The main types of these techniques (medial, lateral, intermediate and transverse) can be combined and should be performed so as to avoid airway dysfunction. A comfortable and reproducible method needs to be established to prevent complications and to ensure morbidity is kept to a minimum $[1,2]$.

Intermediate osteotomies are vertical cuts made between the medial and lateral osteotomies, following the medial and prior to the lateral. They are indicated in upper third deviations, when the contour of nasal bones is excessively convex or concave, or when there is evident disparity in their width. The vertical cut starts inferiorly at the point of maximum convexity/concavity, more or less midway between the medial and lateral osteotomies, with the osteotome in a horizontal position. This osteotomy can also be performed externally [3-5]. The authors try to achieve approximately equal fragments of bone on both sides between the medial and lateral osteotomies. Two independent fragments of bone are then created, which can be reduced independently.

One method that can be employed to analyze the nasal dorsum aesthetically is the evaluation of the eyebrow-tip lines. These lines start superiorly at the supraciliary ridge, make a curve at the intercanthal line (the central part of the eyebrow-tip lines) and continue down to the tip-defining 
points, running parallel to the lateral nasal walls [1]. The distance between the two lines at the keystone area should be $75-80 \%$ of the width of the alar base [1].

This report focuses on a different and more extensive use of intermediate osteotomies. This technique was first performed in patients with typical "crooked noses" in whom the authors have noticed much better aesthetic harmonious results in the contour and shape of the dorsum than in cases where intermediate osteotomies were not carried out.

This manuscript aims to present a different approach to intermediate osteotomies that can be routinely used in selected patients to provide harmony to the nasal contour.

\section{Methods}

During preoperative patient evaluation, the patient's standard history is collected and a physical examination performed. The first visit entails a careful facial and nasal analysis [6], following which photo-documentation is produced; three-dimensional CT scan facial reconstructions are required for every patient to study each case and plan the surgery (Fig. 1). Video-documentation is also routinely generated, since the face and the nose are dynamic structures that, in certain cases, are better evaluated in movement.

The surgical approach presented here entails performing initial medial osteotomies, which are initiated in the midline with a $3 \mathrm{~mm}$ sharp unguarded micro-osteotome placed intranasally at the caudal border of the nasal bones and then angled $15^{\circ}-20^{\circ}$ laterally until the frontal bone is reached. If a bony dorsal hump is to be removed, medial osteotomies are frequently not required, as the open roof allows the nasal bones to be moved medially. Intermediate osteotomies (Figs. 2, 3) start inferiorly at the point of maximum convexity/concavity of the nasal bones, with the
Fig. 1 Three-dimensional CT scan facial reconstructions
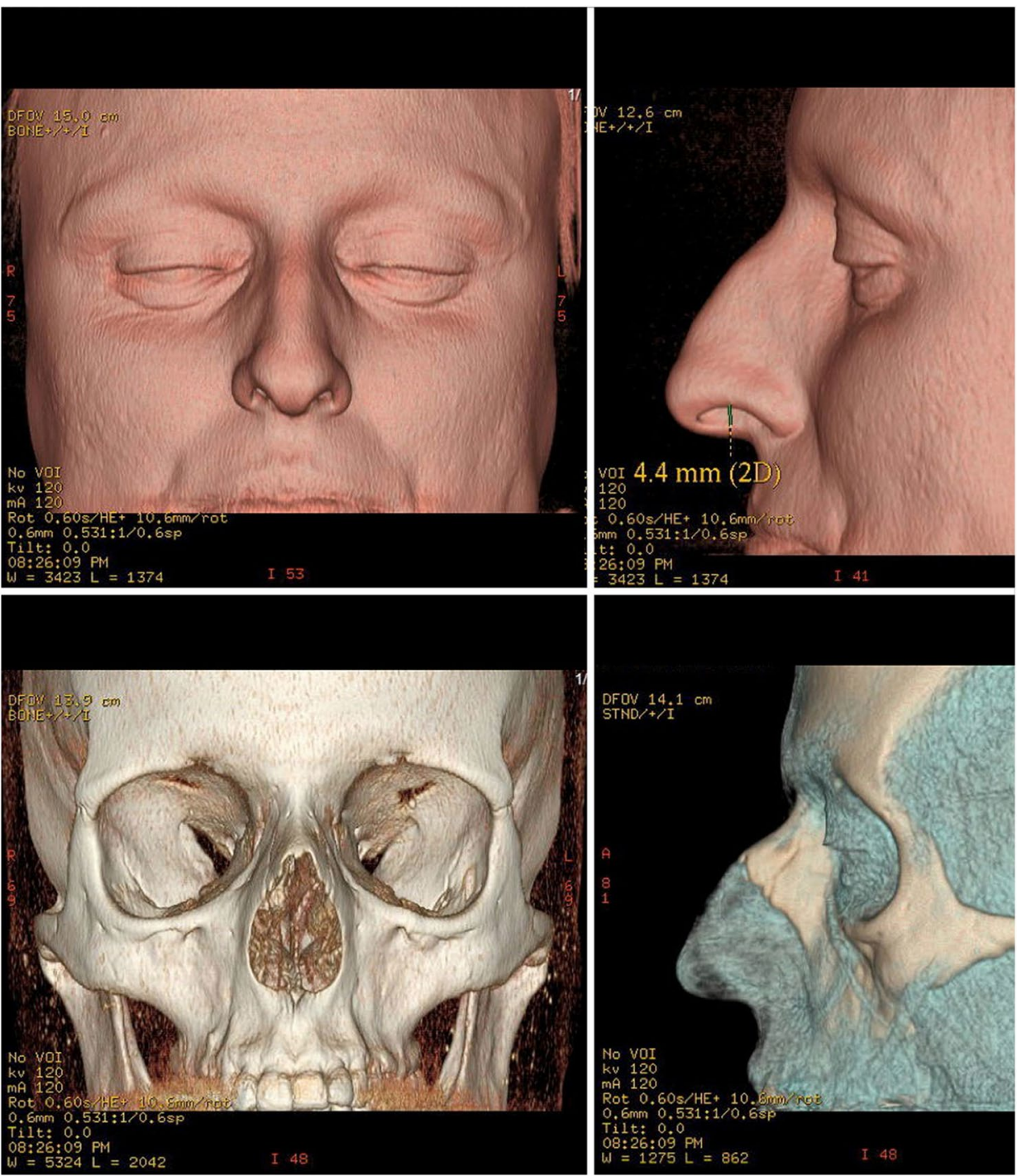
Fig. 2 Medial, intermediate and lateral osteotomies on three-dimensional CT scan facial reconstructions
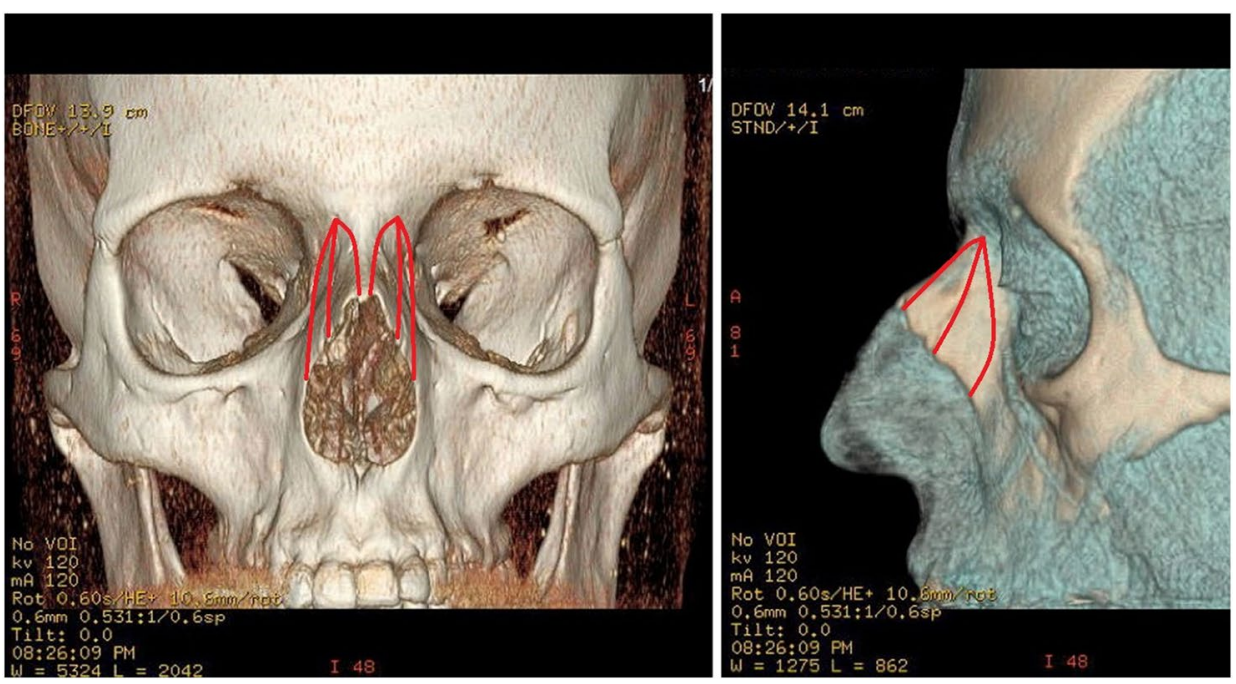

Fig. 3 Removal of a dorsal hump (left); placement of intermediate and lateral osteotomies (middle); effect of intermediate osteotomies-malleable bony fragments (right)

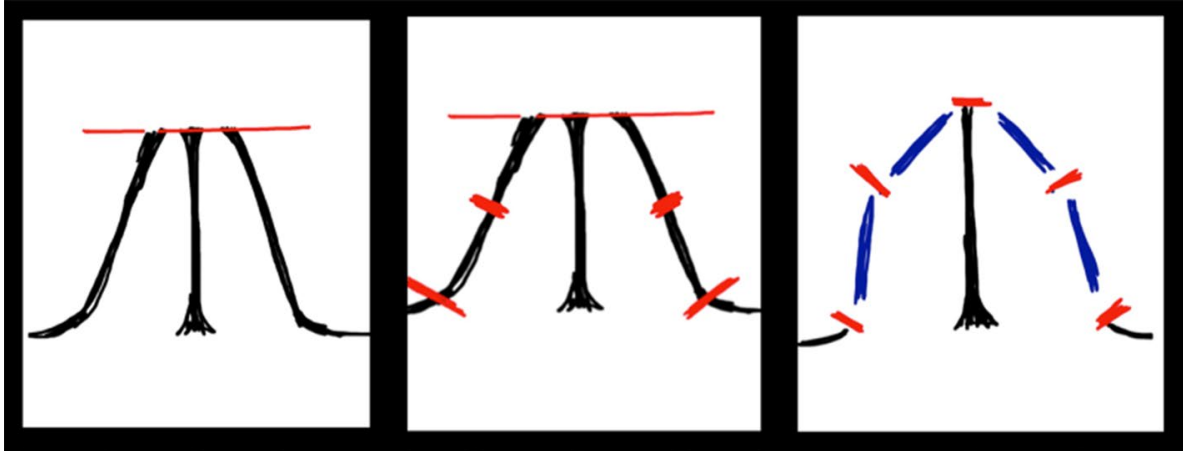

osteotome in a horizontal position. Following removal of a bony hump, intermediate osteotomies start at the midpoint between the medial part of the pyriform aperture still in place and the expected start point of the lateral osteotomies. The osteotome then takes a vertical direction until it encounters the frontal bone and the cephalic end of the medial osteotomies. Lateral osteotomies, initiated at the transition between the nasal sidewall plan and the facial plan, are completed following a high-low-high path, to maintain Webster's triangle intact. An infracture is then made upon completion of the osteotomies of both sides. At this point, the nasal dorsum is molded to achieve the desired result. Depending on the patient, transverse osteotomies may be performed. The authors try to preserve the periosteum in most of the cases.

Over the last few years, intermediate osteotomies have been routinely performed in patients with a change in eyebrow-tip line harmony (narrowed or enlarged distance, asymmetry between the two or irregularities in their curve). These patients were concerned about the aesthetic appearance of their nose. All operations were performed by the senior surgeon (DOC) and all patients were followed during the postoperative period for at least
3 months. Crooked noses and noses with a postoperative filler application were excluded from this study. Taking these criteria into consideration, 50 consecutive patients, who had undergone surgery between January 2014 and June 2015, were selected.

During the preparation of this paper, operative reports and medical charts were reviewed retrospectively to gather details concerning the surgical and postoperative period.

All patients were asked in a telephone interview to evaluate their satisfaction level with the cosmetic outcome of the nasal dorsum on a 5-point visual analog scale (VAS) (5-completely satisfied; 1 -no satisfaction at all with the outcome). They were also asked to compare the impact of nasal obstruction on their quality of life, pre- and postoperatively, by completing the NOSE scale [7, 8]. This survey consists of answering five parameters ("nasal congestion or stuffiness"; "nasal blockage or obstruction"; "trouble breathing through the nose"; "trouble sleeping"; "unable to get enough air through the nose during exercise or exertion") on a 5-point VAS (0- "not a problem"; 4- "severe problem"). The answers are then added and multiplied by 20 to generate possible score out of 100 for analysis. A higher score represents a more severe nasal obstruction. 
A description of the tip and septum surgery is beyond the scope of this report.

This study was approved by the institutional review board. Written informed consent was provided by all patients and no stipend was awarded.

\section{Results}

Between January 2014 and June 2015, 50 patients with disturbances of the eyebrow-tip line harmony met the inclusion criteria and consented to participate in the study. They requested an improvement in dorsum appearance, with or without simultaneous improvement of the nasal tip and/or correction of concomitant nasal obstruction.

The subjective assessment of cosmetic outcome yielded a score of 4.4 (average score, from a maximum of 5), while the NOSE scale generated a score of 227.6 (average score, from a maximum of 2000) preoperatively, and 94.4 postoperatively.

In this report, we also present pictures of three representative cases (Figs. 4, 5, 6). Typical complications of the upper third of the nose following osteotomies, such as rocker deformity, postoperative open roof deformity or greenstick fractures [9], were not observed in any of the 50 patients.

\section{Discussion}

Intermediate osteotomies have only been described in a few publications and are referred to as an indication for upper third deviations, excessively convex or concave nasal bones contour or evident disparity in nasal bone width [3-5]. To date there have been no reports of the routine use of this technique in cases in which a change in eyebrow-tip line harmony exists. In our opinion, nasal dorsum appearance plays a major role in nasal and facial harmony and should not be neglected.

Medial osteotomies are performed first, followed by intermediate and finally the lateral osteotomies. This sequence gives each osteotomy the necessary stability to be completed [4]. The use of a micro-osteotome allows more precise osteotomies to be achieved, while keeping soft tissue damage to a minimum and facilitating correct and stable healing [10].

When lateral osteotomies are required, the authors always perform the high-low-high fashion. This technique, first performed by Webster and Farrior in the 1970s, avoids pyriform aperture and internal nasal valve disturbances and consequent iatrogenic nasal obstruction. Lateral nasal sidewall soft tissue connections are also better maintained [11].

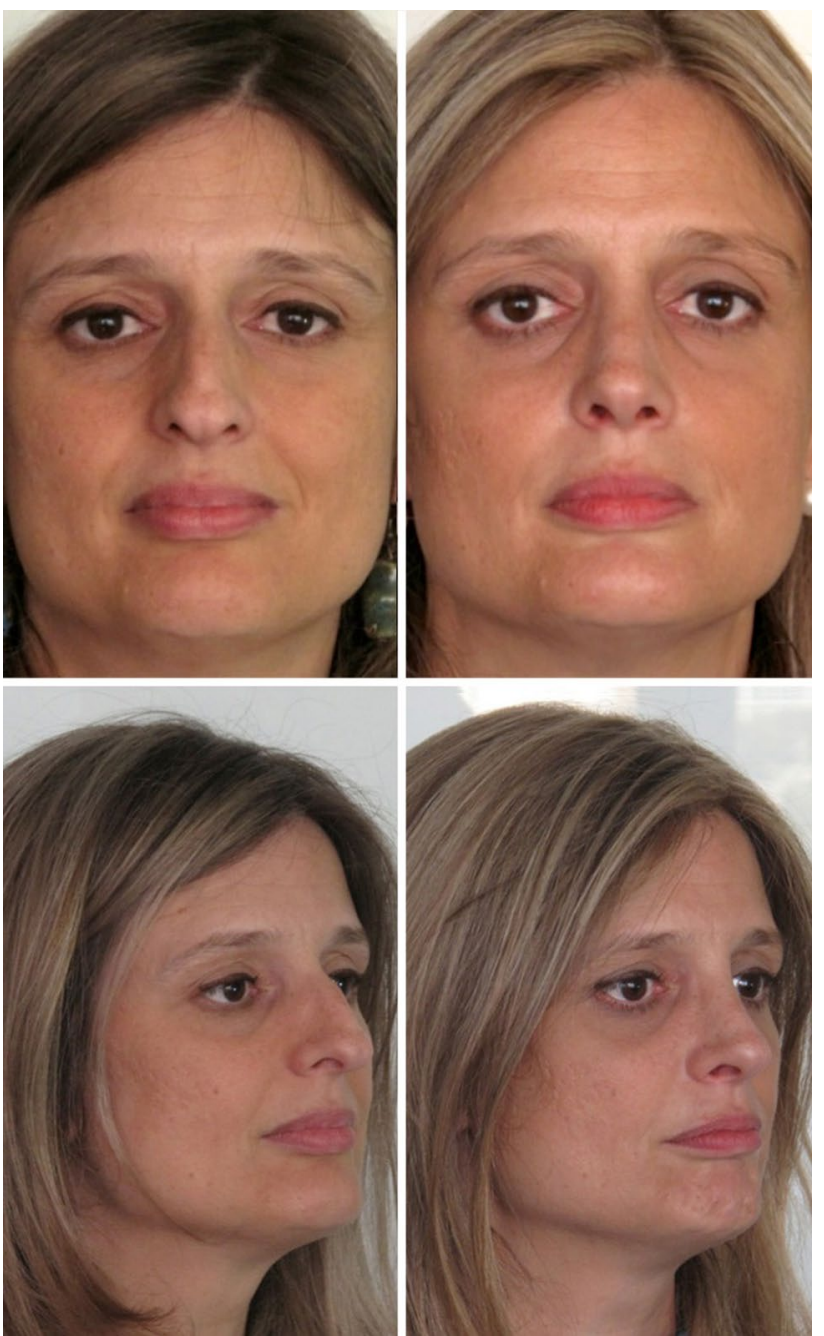

Fig. 4 Preoperative (left) and postoperative (right; 1 year post-surgery) clinical images-patient 1

The satisfaction with the cosmetic outcome of the nasal dorsum was high according to the patients' subjective rating (VAS average score, 4.4), with 54\% of the patients rating it as 5.0 (completely satisfied). The average score of the NOSE scale improved from 227.6 preoperatively to 94.4 postoperatively, demonstrating that intermediate osteotomies, when used routinely and accurately, do not disturb the nasal function.

Intermediate osteotomies, in combination with medial and lateral osteotomies, have the major advantage of providing the nasal surgeon the possibility of avoiding the mobilization of a large bony segment, which is difficult to manipulate correctly, by creating more malleable bony fragments instead. In the hands of the senior surgeon, it is easier to give the nasal dorsum the intended shape, with fewer irregularities, while controlling the internal nasal valve patency. This technique, in combination with nasal dorsum infracture, enables the creation of a smoother and 

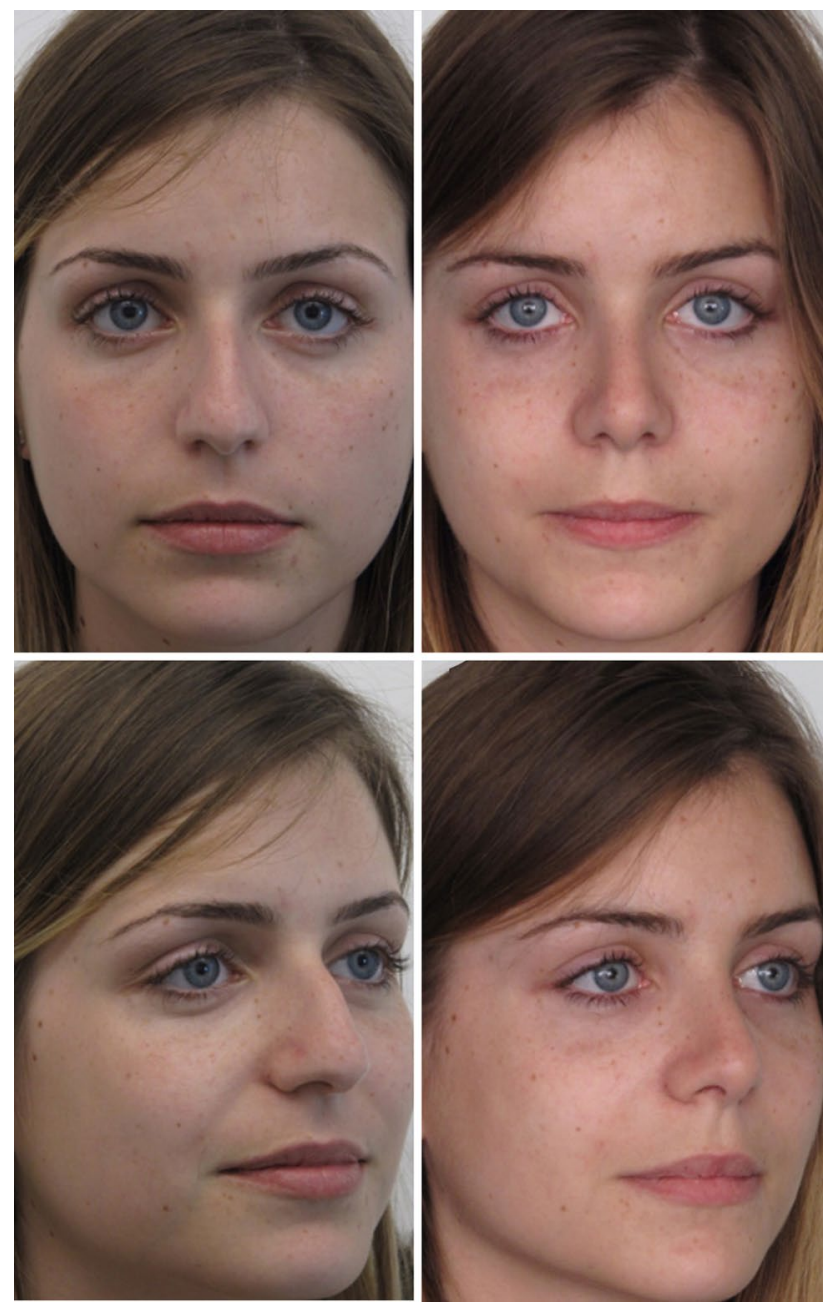

Fig. 5 Preoperative (left) and postoperative (right; 1 year post-surgery) clinical images-patient 2

more natural nasofacial contour and harmonious eyebrowtip lines, maintaining the nasal function undamaged.

Nasal obstruction is prevented using the high-low-high fashion lateral osteotomy, while controlled elevation of soft tissues over the nasal bones prevents the formation of moving bones and nasal collapse. The limitations of this study include the expected disadvantages of a retrospective evaluation.

\section{Conclusion}

This approach, used routinely in patients with aesthetically unpleasant changes in eyebrow-tip line features, allows for a natural, cosmetically agreeable and harmonious dorsum contour to be obtained, while conserving nasal function and achieving high patient satisfaction. This paper does not present a new technique, but instead proposes a novel
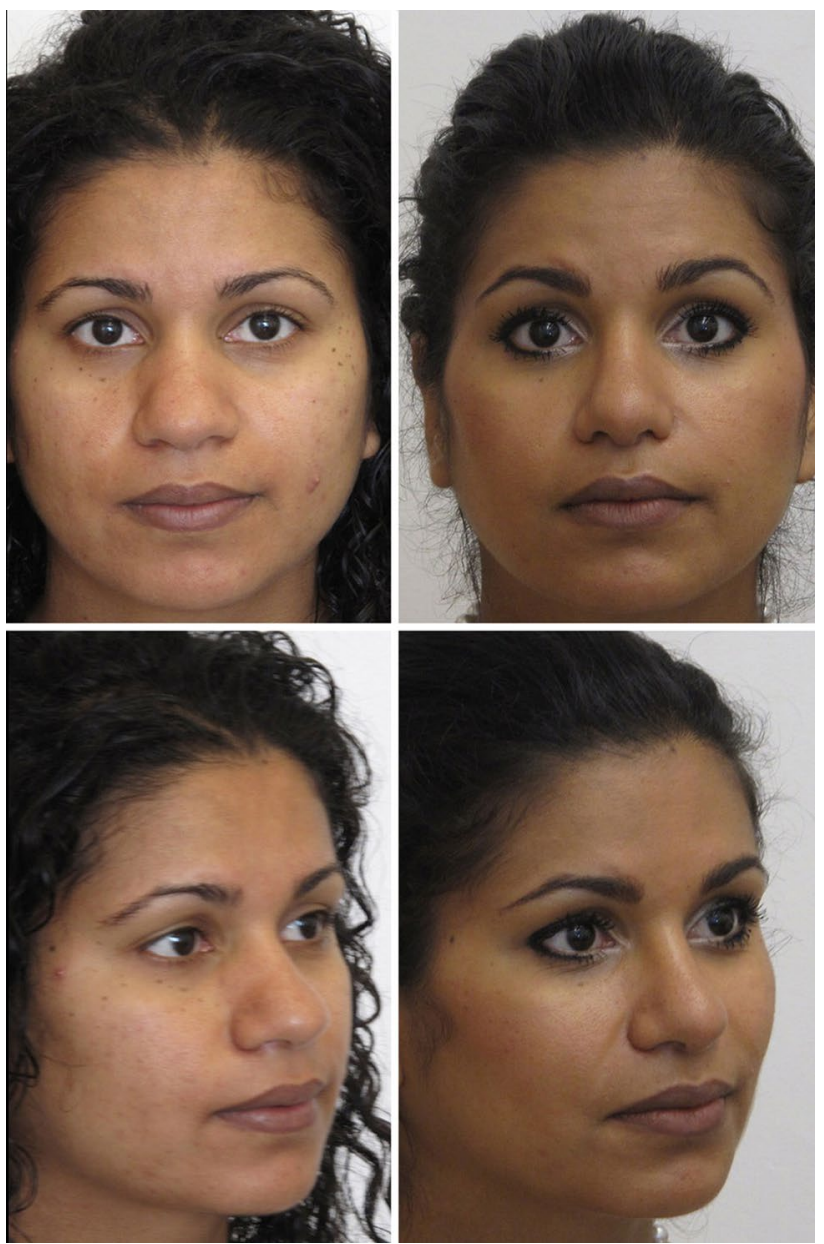

Fig. 6 Preoperative (left) and postoperative (right; 1 year post-surgery) clinical images-patient 3

approach to an already known method that can be routinely used in selected patients as a complement to other rhinoplasty procedures.

Author contributions All authors had full access to all the data in the study and take responsibility for the integrity of the data and the accuracy of the data analysis. Study concept and design: all authors. Acquisition of data: Nunes, Diogo; Tinoco, Catarina; Carmo, Diogo. Analysis and interpretation of data: Nunes, Diogo; Tinoco, Catarina; Carmo, Diogo. Drafting of the manuscript: Nunes, Diogo; Tinoco, Catarina; Carmo, Diogo. Critical revision of the manuscript for important intellectual content: all authors. Statistical analysis: Nunes, Diogo; Tinoco, Catarina; Carmo, Diogo. Administrative, technical, or material support: all authors. Study supervision: Carmo, Diogo; Paço, João.

\section{Compliance with ethical standards}

\section{Conflict of interest None reported.}

Research involving human participants and/or animals All procedures performed in studies involving human participants were in accordance with the ethical standards of the institutional and/or 
national research committee and with the 1964 Helsinki declaration and its later amendments or comparable ethical standards; this article does not contain any studies with animals performed by any of the authors.

Informed consent Informed consent was obtained from all individual participants included in the study.

\section{References}

1. Gunter J, Rohrich R, Adams W (2007) Dallas rhinoplasty: nasal surgery by the masters. Quality Medical Publishing, Inc., St. Louis

2. Zoumalan R, Shah A, Constantinides M (2010) Quantitative comparison between microperforating osteotomies and continuous lateral osteotomies in rhinoplasty. Arch Facial Plast Surg 12(2):92-96

3. Behrbohm H, Tardy ME (2004) Essentials of septorhinoplasty: philosophy-approaches-techniques. Thieme, Stuttgart

4. Gerarchi P, Mendelsohn M (2007) The wide nasal dorsum: evaluation and management. Otolaryngol Head Neck Surg 136:S32-S40
5. Shah A, Constantinides M (2006) Aligning the bony nasal vault in rhinoplasty. Facial Plast Surg 22:3-8

6. Prendiville S, Zimbler M, Kokoska M, Thomas R (2002) Middle-vault narrowing in the wide nasal dorsum: the "reverse spreader" technique. Arch Facial Past Surg 4:52-55

7. Stewart MG, Witsell DL, Smith TL, Weaver EM, Yueh B, Hannley MT (2004) Development and validation of the nasal obstruction symptom evaluation (NOSE) scale. Otolaryngol Head Neck Surg 130(2):157-163

8. Rhee J, Sullivan C, Frank D, Kimbell J, Garcia J (2014) A systematic review of patient-reported nasal obstruction scoresdefining normative and symptomatic ranges in surgical patients. JAMA. Facial Plast Surg 16(3):219-225

9. Papel I et al (2016) Facial plastic and reconstructive surgery. Thieme, Stuttgart

10. Tardy ME, Denney JC (1984) Micro-osteotomies in rhinoplasty. Facial Plast Surg 1:137-145

11. Byrne P, Walsh W, Hilger P (2003) The use of "inside-out" lateral osteotomies to improve outcome in rhinoplasty. Arch Facial Past Surg 5:251-255 